\title{
GENUS 2 CURVES THAT ADMIT A DEGREE 5 MAP TO AN ELLIPTIC CURVE
}

\author{
K. MAGAARD, T. SHASKA, AND H. VÖLKLEIN
}

\begin{abstract}
We continue our study of genus 2 curves $C$ that admit a cover $C \rightarrow E$ to a genus 1 curve $E$ of prime degree $n$. These curves $C$ form an irreducible 2-dimensional subvariety $\mathcal{L}_{n}$ of the moduli space $\mathcal{M}_{2}$ of genus 2 curves. Here we study the case $n=5$. This extends earlier work for degree 2 and 3, aimed at illuminating the theory for general $n$.

We compute a normal form for the curves in the locus $\mathcal{L}_{5}$ and its three distinguished subloci. Further, we compute the equation of the elliptic subcover in all cases, give a birational parametrization of the subloci of $\mathcal{L}_{5}$ as subvarieties of $\mathcal{M}_{2}$ and classify all curves in these loci which have extra automorphisms.
\end{abstract}

\section{INTRODUCTION}

We continue our study of genus 2 curves $C$ that admit a cover $C \rightarrow E$ to a genus 1 curve $E$. We assume the degree $n$ of the cover is a prime. For $n=2,3$ such curves $C$ already occur in the work of Hermite, Goursat, Burkhardt, Brioschi, and Bolza, see Krazer [15] (p. 479). For general $n$, they have been studied by Frey, Kani and others in [3, 4, 5, 13, 24, 26, 23, 17]. The following can be found in these references. These curves $C$ are parametrized by an irreducible 2-dimensional subvariety $\mathcal{L}_{n}$ of the moduli space $\mathcal{M}_{2}$ of genus 2 curves. If $C$ corresponds to a generic point of $\mathcal{L}_{n}$ then $C$ admits exactly two degree $n$ covers $C \rightarrow E_{1}$ and $C \rightarrow E_{2}$ to a genus 1 curve, up to equivalence. Here we call two such covers equivalent if they correspond to the same elliptic subfield of the function field of $C$. The Jacobian of $C$ is isogenous to $E_{1} \times E_{2}$; see 22 for details.

The degree $n$ cover $\psi: C \rightarrow E$ induces a degree $n$ cover $\phi: \mathbb{P}^{1} \rightarrow \mathbb{P}^{1}$ such that the following diagram commutes

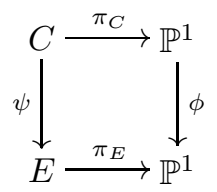

Figure 1. The basic setup

Here $\pi_{C}: C \rightarrow \mathbb{P}^{1}$ and $\pi_{E}: E \rightarrow \mathbb{P}^{1}$ are the natural degree 2 covers. Let $r$ be the number of branch points of the cover $\phi: \mathbb{P}^{1} \rightarrow \mathbb{P}^{1}$. Then $r=4$ or $r=5$, with $r=5$

Date: July 9, 2018.

2000 Mathematics Subject Classification. Primary: 14H10, 14H30, Secondary: 14H45, 14H35.

Key words and phrases. genus 2 curves, Hurwitz spaces, Humbert surfaces, covers of curves.

The first author was partially supported by the NSA. 
being the generic case and $r=4$ occurring for a certain 1-dimensional sub-locus of $\mathcal{L}_{n}$. We refer to the case $r=5$ (resp., $r=4$ ) as the non-degenerate case, resp., the degenerate case; see [5] or Theorem 3.1 in [22].

Here we study the case $n=5$. This extends earlier work for $n=2,3$ in Shaska 22], 23], and Shaska/Völklein [26]. So from now on we assume $n=5$. Then the cover $\phi: \mathbb{P}^{1} \rightarrow \mathbb{P}^{1}$ has one of the following ramification structures:

$\begin{array}{ccc}\begin{array}{c}\text { non-degenerate: } \\ \text { degenerate: }\end{array} & & \left((2)^{2},(2)^{2},(2)^{2},(2),(2)\right) \\ & \text { I) } & \left((2)^{2},(2)^{2},(4),(2)\right) \\ & \text { II) } & \left((2)^{2},(2)^{2},(2) \cdot(3),(2)\right) \\ \text { III }) & \left((2)^{2},(2)^{2},(2)^{2},(3)\right)\end{array}$

TABLE 1. ramification structure of $\phi$

see [5] or 22] for ramification structures of arbitrary degree. This data lists the ramification indices $>1$ over the branch points. E.g., in the last case there is one branch point that has exactly one ramified point over it, of index 3 , and each of the other 3 branch points has exactly two ramified points over it, of index 2 .

The main feature that distinguishes the case $n=5$ from all other values $n>5$ is that the cover $\phi$ does not determine $\psi: C \rightarrow E$ uniquely, but there is essentially two choices of $\psi$ for a given $\phi$. These two choices correspond to the two branch points of $\phi$ of ramification structure (2) (notation as in Table 1) - anyone of these two branch points can be chosen to ramify in $E$ while the other doesn't. This phenomenon implies that the function field of $\mathcal{L}_{5}$ is a quadratic extension of the function field of the Hurwitz space parameterizing the covers $\phi$.

We show that each of the 3 degenerate cases corresponds to an irreducible 1dimensional locus on $\mathcal{L}_{5}$, two of which have genus zero and one has genus 1 . We compute a normal form for the curves in the locus $\mathcal{L}_{5}$ and its three distinguished sub-loci. We give a bi-rational parametrization of these sub-loci as subvarieties of $\mathcal{M}_{2}$ and classify all curves in them that have extra automorphisms.

A few remarks on computations: The computations of this paper were performed using Maple or Mathematica. While we provide a sketch of such computations, we skip most of the details. We are assuming that the reader is familiar with some computational algebra packages and knows the basic methods of "solving" systems of non-linear equations (i.e., Groebener bases algorithms, resultants, etc). Throughout the paper we use the terms "computations are easy" or "straightforward". This should not be confused with "fast" or "quick". Some of these computations took several days. For the interested reader who wants to re-produce such results we provide details on [25]. The explicit equations of the loci $\mathcal{Y}_{i}, i=1,2,3$ or the list of genus two curves with extra automorphisms (cf. Section 4) which are in the locus $\mathcal{Y}_{i}$ can be provided by the second author upon request.

\section{Background on Hurwitz spaces and Humbert surfaces}

2.1. Hurwitz spaces of covers $\phi: \mathbb{P}^{1} \rightarrow \mathbb{P}^{1}$. Two covers $f: X \rightarrow \mathbb{P}$ and $f^{\prime}:$ $X^{\prime} \rightarrow \mathbb{P}$ are called weakly equivalent if there is a homeomorphism $h: X \rightarrow X^{\prime}$ and an analytic automorphism $g$ of $\mathbb{P}$ (i.e., a Moebius transformation) such that 
$g \circ f=f^{\prime} \circ h$. The covers $f$ and $f^{\prime}$ are called equivalent if the above holds with $g=1$.

Consider a cover $f: X \rightarrow \mathbb{P}$ of degree $n$, with branch points $p_{1}, \ldots, p_{r} \in \mathbb{P}$. Pick $p \in \mathbb{P} \backslash\left\{p_{1}, \ldots, p_{r}\right\}$, and choose loops $\gamma_{i}$ around $p_{i}$ such that $\gamma_{1}, \ldots, \gamma_{r}$ is a standard generating system of the fundamental group $\Gamma:=\pi_{1}\left(\mathbb{P} \backslash\left\{p_{1}, \ldots, p_{r}\right\}, p\right.$ ) (see [28, Thm. 4.27); in particular, we have $\gamma_{1} \cdots \gamma_{r}=1$. Such a system $\gamma_{1}, \ldots, \gamma_{r}$ is called a homotopy basis of $\mathbb{P} \backslash\left\{p_{1}, \ldots, p_{r}\right\}$. The group $\Gamma$ acts on the fiber $f^{-1}(p)$ by path lifting, inducing a transitive subgroup $G$ of the symmetric group $S_{n}$ (determined by $f$ up to conjugacy in $S_{n}$ ). It is called the monodromy group of $f$. The images of $\gamma_{1}, \ldots, \gamma_{r}$ in $S_{n}$ form a tuple of permutations $\sigma=\left(\sigma_{1}, \ldots, \sigma_{r}\right)$ called a tuple of branch cycles of $f$.

We say a cover $f: X \rightarrow \mathbb{P}$ of degree $n$ is of type $\sigma$ if it has $\sigma$ as tuple of branch cycles relative to some homotopy basis of $\mathbb{P}$ minus the branch points of $f$. Let $\mathcal{H}_{\sigma}$ be the set of weak equivalence classes of covers of type $\sigma$. The Hurwitz space $\mathcal{H}_{\sigma}$ carries a natural structure of an quasiprojective variety (see [6], 28] ).

We have $\mathcal{H}_{\sigma}=\mathcal{H}_{\tau}$ if and only if the tuples $\sigma, \tau$ are in the same braid orbit $\mathcal{O}_{\tau}=\mathcal{O}_{\sigma}$ (see 28], Def. 9.3 or [19]). In the case of the covers $\phi: \mathbb{P}^{1} \rightarrow \mathbb{P}^{1}$ from above, the corresponding braid orbit consists of all tuples in $S_{5}$ whose cycle type matches the ramification structure of $\phi$. This and the genus of $\mathcal{H}_{\sigma}$ in the degenerate cases (see the following table) has been computed by the BRAID PACKAGE; see [19.

\begin{tabular}{c|c|c|c|c|c} 
Case & cycle type of $\sigma$ & $\#\left(\mathcal{O}_{\sigma}\right)$ & $G$ & $\operatorname{dim} \mathcal{H}_{\sigma}$ & genus of $\mathcal{H}_{\sigma}$ \\
\hline & $\left(2^{2}, 2^{2}, 2^{2}, 2,2\right)$ & 40 & $S_{5}$ & 2 & - \\
& & & & & \\
I) & $\left(2^{2}, 2^{2}, 4,2\right)$ & 8 & $S_{5}$ & 1 & 0 \\
II $)$ & $\left(2^{2}, 2^{2}, 2 \cdot 3,2\right)$ & 6 & $S_{5}$ & 1 & 0 \\
III) & $\left(2^{2}, 2^{2}, 2^{2}, 3\right)$ & 9 & $A_{5}$ & 1 & 1
\end{tabular}

TABLE 2. Hurwitz spaces and their dimensions

In the next section we compute a normal form for the curve $C$ in $\mathcal{L}_{5}$ and each of its degenerates subloci. Further, we find birational parametrizations for each degenerate sublocus of $\mathcal{L}_{5}$. The non-degenerate case is a little more complicated computationally; we omit the details.

2.2. Humbert surfaces. Let $\mathcal{A}_{2}$ denote the moduli space of principally polarized abelian surfaces. It is well known that $\mathcal{A}_{2}$ is the quotient of the Siegel upper half space $\mathfrak{H}_{2}$ of symmetric complex $2 \times 2$ matrices with positive definite imaginary part by the action of the symplectic group $S p_{4}(\mathbb{Z})$; see [7] (p. 211).

Let $\Delta$ be a fixed positive integer and $N_{\Delta}$ be the set of matrices

$$
\tau=\left(\begin{array}{cc}
z_{1} & z_{2} \\
z_{2} & z_{3}
\end{array}\right) \in \mathfrak{H}_{2}
$$

such that there exist nonzero integers $a, b, c, d, e$ with the following properties:

$$
\begin{aligned}
& a z_{1}+b z_{2}+c z_{3}+d\left(z_{2}^{2}-z_{1} z_{3}\right)+e=0 \\
& \Delta=b^{2}-4 a c-4 d e
\end{aligned}
$$


The Humbert surface $\mathcal{H}_{\Delta}$ of discriminant $\Delta$ is called the image of $N_{\Delta}$ under the canonical map

$$
\mathfrak{H}_{2} \rightarrow \mathcal{A}_{2}:=S p_{4}(\mathbb{Z}) \backslash \mathfrak{H}_{2},
$$

see [10], 2] or [21] for details. It is known that $\mathcal{H}_{\Delta} \neq \emptyset$ if and only if $\Delta>0$ and $\Delta \equiv 0$ or $1 \bmod 4$. Humbert (1900) studied the zero loci in Eq. (11) and discovered certain relations between points in these spaces and certain plane configurations of six lines; see [10, for more details.

For a genus 2 curve $C$ defined over $\mathbb{C},[C]$ belongs to $\mathcal{L}_{n}$ if and only if the isomorphism class $\left[J_{C}\right] \in \mathcal{A}_{2}$ of its (principally polarized) Jacobian $J_{C}$ belongs to the Humbert surface $\mathcal{H}_{n^{2}}$, viewed as a subset of the moduli space $\mathcal{A}_{2}$ of principally polarized abelian surfaces; see [21] (Theorem 1, pg. 125) for the proof of this statement. In particular, every point in $\mathcal{H}_{n^{2}}$ can be represented by an element of $\mathfrak{H}_{2}$ of the form

$$
\tau=\left(\begin{array}{cc}
z_{1} & \frac{1}{n} \\
\frac{1}{n} & z_{2}
\end{array}\right), \quad z_{1}, z_{2} \in \mathfrak{H} .
$$

There have been many attempts to explicitly describe these Humbert surfaces. For some small discriminant this has been done by several authors; see [26, [23], [17. Geometric characterizations of such spaces for $\Delta=4,8,9$, and 12 were given by Humbert (1900) in [10] and for $\Delta=13,16,17,20,21$ by Birkenhake/Wilhelm (2003) in 2]. The Humbert surface of discriminant 25 has not been explicitly described.

\section{Parametrization of the Covers $\phi$}

In Theorem 1, ii) we give an explicit equation for the cover $\phi: \mathbb{P}^{1} \rightarrow \mathbb{P}^{1}$ associated with any degree 5 cover $C \rightarrow E$ (as in the Introduction). It is easier to formulate the result in terms of function fields as follows:

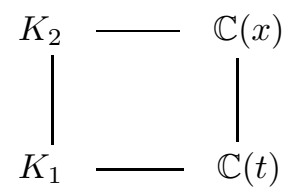

Theorem 1. Let $K_{2}$ be a genus 2 function field over $\mathbb{C}$ and $K_{1}$ a genus 1 subfield with $\left[K_{2}: K_{1}\right]=5$. Then there are $x, t \in K_{2}$, unique up to the action of $S_{3}$ given by the transformations

$$
(x, t) \mapsto\left(\frac{1}{x}, \frac{1}{t}\right), \quad(x, t) \mapsto(1-x, 1-t),
$$

and $a, b \in \mathbb{C} \backslash\{0\}, a+b \neq-1$, unique up to the action of $S_{3}$ given by the transformations

$$
(a, b) \mapsto\left(\frac{a}{b}, \frac{1}{b}\right), \quad(a, b) \mapsto(a,-a-b-1)
$$

such that the following holds:

i) $\mathbb{C}(x)$ is the unique rational subfield of $K_{2}$ of degree 2. The generator $\iota$ of $\operatorname{Gal}\left(K_{2} / \mathbb{C}(x)\right)$ is called the hyperelliptic involution of $K_{2}$ and it fixes $K_{1}$. 
ii) $\mathbb{C}(t)=K_{1} \cap \mathbb{C}(x)$ and

$$
\begin{aligned}
& t=\varphi(x)=x\left(\frac{F_{1}(x)}{F_{2}(x)}\right)^{2} \\
& t-1=(x-1)\left(\frac{F_{3}(x)}{F_{2}(x)}\right)^{2}
\end{aligned}
$$

where

$$
\begin{aligned}
& F_{1}(x)=x^{2}+\left(2 a+2 b+a^{2}\right) x+2 a b+b^{2} \\
& F_{2}(x)=(2 a+1) x^{2}+\left(a^{2}+2 a b+2 b\right) x+b^{2} \\
& F_{3}(x)=x^{2}-\left(a^{2}-2 b\right) x+b^{2}
\end{aligned}
$$

Proof. i) is well-known (see e.g. [5]).

Since $\iota$ fixes $K_{1}$, the field $K_{1} \cap \mathbb{C}(x)$ is a subfield of $K_{1}$ of degree 2 . It is of the form $\mathbb{C}(t)$, where $t=\varphi(x)$ is a rational function of $x$ of the ramification structure described in Table 1 . We normalize $t$ by assuming that $\varphi$ is ramified at $t=0,1, \infty$ of type not equal to (2) or (3) (notation as in Table 1). Similarly we normalize $x$ by assuming that $x=0,1, \infty$ is that place over $t=0,1, \infty$, respectively, that is unramified except in the case that there is no unramified place over the corresponding value of $t$ in which case we assume that it has ramification index 3 (The latter occurs only in the degenerate case II). From Table 1 we see that this normalization determines $x$ and $t$ uniquely up to the action of the subgroup of $P G L_{2}(\mathbb{C})$ permuting $0,1, \infty$. Indeed, the three places that we assign the values $0,1, \infty$ are determined uniquely by $K_{1}$ and $K_{2}$, but we have to order the three places by assigning these values and all the possible orderings are conjugate under the group $S_{3}$ from above. This proves the uniqueness assertion on $x, t$.

From the ramification structure of $\varphi$ and the above normalisations it follows that $\varphi(X) / X$ and $(\varphi(X)-1) /(X-1)$ are squares in $\mathbb{C}(X)$ (where $X$ is a variable). Thus

$$
\varphi(X)=X \frac{\left(X^{2}+M X+N\right)^{2}}{\left(A X^{2}+B X+C\right)^{2}}
$$

and the condition $\varphi(1)=1$ implies that $1+M+N= \pm(A+B+C)$. Replacing $A, B, C$ by their negatives if necessary we can in any case write $\varphi(X)$ in the form

$$
\varphi(X)=X \frac{\left(X^{2}+M X+A+B+C-M-1\right)^{2}}{\left(A X^{2}+B X+C\right)^{2}} .
$$

Factoring $\varphi(X)-1$ now yields an expression of the form

$$
\varphi(X)-1=(X-1) \frac{g(X)}{\left(A X^{2}+B X+C\right)^{2}}
$$

where $g(X)$ is a monic polynomial of degree 4. We know that $g(X)$ has to be a square. To exploit this condition, we observe that there is a simple criterion for a monic degree 4 polynomial to be a square, see Remark 1 below. Applying this criterion to $g(X)$ yields

$$
\begin{aligned}
& 2 M-8 A-8 B-8 C+8 A B+16 A C+11 A^{2}+8 B^{2}+4 M A^{2}+8 M^{2} A^{2}- \\
& 6 M A^{4}-8 A^{2} C+8 A^{3} B-8 A^{2} B-16 M A B+A^{4}-8 A^{3}+A^{6}+3=0
\end{aligned}
$$


and

$$
\begin{aligned}
& (A-1)\left(-A^{3}-A^{2}+4 A M-3 A-8 B+5+4 M\right)\left(-4 M+4 M A^{2}-A^{4}-2 A^{2}\right. \\
& -8 A B+8 A+8 B+16 C-5)=0
\end{aligned}
$$

If $A=1$, then the equation Eq. (6) reduces to $B=M$, which implies that $\varphi(X)=X$ : a contradiction. If the last factor in equation Eq. (7) vanishes, then from Eq. (6) we compute that the resultant of $X^{2}+M X+N$ and $A X^{2}+B X+C$ vanishes. Hence $\varphi(X)$ has degree $\leq 3$, again a contradiction. Therefore, Eq. (7) reduces to

$$
-A^{3}-A^{2}+4 A M-3 A-8 B+5+4 M=0 .
$$

From this we get

$$
B=\frac{1}{8}\left(-A^{3}-A^{2}-3 A+4 A M+4 M+5\right) .
$$

Plugging this into Eq. (6) we get

$$
C=\frac{1}{64}\left(A^{2}+2 A-4 M-3\right)^{2}
$$

Now we define $a, b$ as follows

$$
a=\frac{A-1}{2}, \quad b=\frac{1}{8}\left(-A^{2}+6 A-4 M-5\right)
$$

One can easily check that the formulas in ii) hold. Furthermore, $a \neq 0$ because $A \neq 1$ as noted before. Also, $b \neq 0$ and $a+b \neq-1$ because otherwise the resultant of $F_{1}(X)$ and $F_{2}(X)$ vanishes (which is a contradiction because then $\operatorname{deg} \varphi(X) \leq 3$ ).

It remains to prove the uniqueness assertion on $a, b$. Clearly, $a, b$ are uniquely determined by $\varphi(X)$, hence by $x, t$. Therefore $a, b$ are unique up to the action of $S_{3}$ as in Eq. (2). The induced action on $\varphi(X)$ is generated by the transformations

$$
\varphi(X) \mapsto \frac{1}{\varphi(1 / X)} \quad \text { and } \quad \varphi(X) \mapsto 1-\varphi(1-X) .
$$

From this we compute that $S_{3}$ acts on $a, b$ as in Eq. (3).

Remark 1. The polynomial

$$
X^{4}+\alpha X^{3}+\beta X^{2}+\gamma X+\delta
$$

is a square in $\mathbb{C}(X)$ if and only if

$$
8 \gamma=\alpha\left(4 \beta-\alpha^{2}\right) \quad \text { and } \quad 64 \delta=\left(4 \beta-\alpha^{2}\right)^{2}
$$

The proof is by direct computation.

Remark 2. (Significance of the $S_{3}$ action)

Let $\varphi(X)$ as in Theorem 1 and let $\varphi_{1}(X)$ be another function of the same shape. Then the corresponding covers $\mathbb{P}^{1} \rightarrow \mathbb{P}^{1}$ are equivalent if and only if $\varphi=\varphi_{1}$. And the covers are weakly equivalent if and only if $\varphi$ and $\varphi_{1}$ are conjugate under the $S_{3}$ action from $E q$. (8). The induced $S_{3}$ action on the parameters $a, b$ is given in Theorem 1. We compute the fixed field of $S_{3}$ in $\mathbb{C}(a, b)$ to be $\mathbb{C}(u, v)$ where

$$
u=\frac{2 a\left(a b+b^{2}+b+a+1\right)}{b(a+b+1)}, \quad v=\frac{a^{3}}{b(a+b+1)} .
$$


Thus the Hurwitz space classifying (up to weak equivalence) the covers $\phi$ of nondegenerate type is birationally parameterized by $u$ and $v$. And the function field of $\mathcal{L}_{5}$ is a quadratic extension of $\mathbb{C}(u, v)$, as we will see later.

Theorem 2. In the situation of Theorem 1, normalize $x, t$ further by assuming that if one of the degenerate cases I) or II) of Table 1 occurs then the exceptional ramification of $\mathbb{C}(x) / \mathbb{C}(t)$ occurs over $t=1$. Then the polynomial

$$
F_{4}(X)=(2 a+1) X^{2}+\left(2 b-2 b a-2 a-a^{2}\right) X+b^{2}+2 a b
$$

has a root $z \neq 1$ and the genus 2 field $K_{2}$ is generated by $x$ together with another element $y$ satisfying

$$
y^{2}=x(x-1) g_{3}(x),
$$

where $g_{3}(x)$ is given in Eq. (10) below. The polynomial $g_{3}(x)$ has coefficients in $\mathbb{C}[a, b, z]$.

Here are the coefficients of $g_{3}(x):=a_{3} x^{3}+a_{2} x^{2}+a_{1} x+a_{0}$ :

$$
\begin{aligned}
a_{0}= & -b^{4}\left(2 b^{3} a+4 b^{3}-2 z a b^{2}+7 b^{2} a^{2}+8 z b^{2}+4 b^{2}+16 a b^{2}+16 z b a+6 a^{3} b+8 b a\right. \\
& \left.+2 z a^{2} b+12 z b+16 b a^{2}+13 z a^{2}+z a^{4}+6 z a^{3}+4 z+12 y a\right) \\
a_{1}= & -b^{2}\left(12 b^{3}+12 b^{4} a+32 z b a-6 a^{4} b^{2}+44 b^{2} a^{3}+6 b a^{2}+24 a b^{2}+10 a^{3} b+44 b^{3} a^{2}+2 b a\right. \\
& +52 b^{3} a+61 b^{2} a^{2}-12 b a^{5}-7 z a^{2}-2 z a+12 z b-4 a^{6}+12 b^{4}-a^{4}-40 z a^{3} b^{2}-16 z b^{3} a^{2} \\
& -12 z a^{5}+36 z b^{2}-18 z a^{3}-26 z a^{4}+56 z a b^{2}+4 a z b^{3}+2 z a^{2} b^{2}-20 z a^{3} b+28 z a^{2} b \\
& \left.+2 z a^{6}+24 z b^{3}+4 z b a^{5}-4 a^{5}-32 z a^{4} b\right) \\
a_{2}= & 5 b^{2} a^{6}+20 b^{2} a^{5}+8 b a^{6}-61 b^{4} a^{2}-18 b^{5} a-56 b^{4} a+4 z b a+5 a^{4} b^{2}-18 b^{2} a^{3}-24 z b^{4} \\
& -14 z b^{4} a-4 a b^{2}+8 b^{3} a^{4}+2 b^{3} a^{5}-54 b^{3} a^{3}-70 b^{3} a^{2}-24 b^{3} a-14 b^{2} a^{2}+4 a^{4} b+10 b a^{5} \\
& -6 z a^{7}+64 z a^{3} b^{3}+38 z a^{4} b^{2}+54 z a^{3} b^{2}+12 z b^{3} a^{2}-14 z a^{6} b-10 z b^{2} a^{5}-4 z a^{7} b-4 a^{6} z b^{2} \\
& +32 a^{2} b^{4} z+2 a^{7} b-z a^{8}-36 z b^{3}-12 z a^{5}-12 z b^{2}-4 z a^{4}-28 z a b^{2}-64 a z b^{3}-5 z a^{2} b^{2} \\
& +16 z a^{2} b+28 z a^{4} b-4 z b a^{5}-13 z a^{6}-12 b^{5}-12 b^{4}+34 z a^{3} b \\
a_{3}= & (2 a+1)\left(z a^{4}-2 a^{3} b+4 z a^{3}+6 z a^{3} b-4 b a^{2}+12 z a^{2} b^{2}+10 z a^{2} b-9 b^{2} a^{2}+5 z a^{2}\right. \\
& \left.-2 b a+2 z a-8 a b^{2}-12 b^{3} a+8 a z b^{3}-4 b^{3}-4 z b-4 b^{4}-12 z b^{2}-8 z b^{3}\right) \\
&
\end{aligned}
$$

Proof. The derivative of $\varphi(X)$ factors as follows:

$$
\varphi^{\prime}(X)=\frac{F_{1}(X) \cdot F_{3}(X) \cdot F_{4}(X)}{F_{2}^{3}(X)}
$$

If $F_{4}(X)$ has no root $z \neq 1$ then $\mathbb{C}(x) / \mathbb{C}(t)$ is unramified outside $t=0,1, \infty$. This contradicts Table 1 . Thus there is a root $z \neq 1$ of $F_{4}(X)$. Furthermore, $\operatorname{deg} F_{4}(X)=2$, because if $2 a+1=0$ then $\operatorname{deg} F_{2}(X)=1$ and degenerate case II) from Table 1 occurs with exceptional ramification over $t=\infty$. But this is excluded in the theorem.

The numerator of $\varphi(X)-\varphi(z)$ is a polynomial $G(X, z)$ of degree 5 in $X$ as well as in $z$. As a polynomial in $X$ it has $X=z$ as a double root because $\varphi^{\prime}(z)=0$. Using the equation $F_{4}(z)=0$ we can re-write every polynomial in $X$ and $z$ such that it becomes linear in $z$. Thus

$$
G(X, z)=(X-z)^{2} \cdot(A(x) z+B(x))
$$


To find $A(x)$ and $B(x)$ we re-write both sides of Eq. (12) so that they become linear in $z$, and then we compare the $z^{1}$-coefficient and the $z^{0}$-coefficient on both sides. The result is displayed in Eq. (10). It follows from [5], that $K_{2}=\mathbb{C}(x, y)$ with $x$ and $y$ satisfying Eq. (9).

Remark 3. In the situation of Theorem 1, assume that none of the degenerate cases I) or II) of Table 1 occurs. Then,

$$
\begin{array}{r}
\Delta(a, b)=(a+b+1) b(2 a+1)(a-b-1)\left(a^{2}-4 b\right)\left(4 b+4+4 a+a^{2}\right) \\
\left(4 b^{2}+4 b+4 b a+a^{2}\right)\left(a^{3}-2 b-2 b a-2 b^{2}\right)(2 a+b) \neq 0
\end{array}
$$

The above assumption implies $F_{4}(1) \neq 0$, thus $z$ from Theorem 2 can be any root of $F_{4}(X)$. The discriminant of the right hand side of the Eq. (9) is non-zero. We compute that discriminant as a polynomial in $a, b, z$. Taking the resultant of this polynomial and $F_{4}(z)$ with respect to $z$ yields $\Delta$ as in Eq. (13). Therefore, $\Delta \neq 0$.

Remark 4. It follows from Theorem 2 that the function field of locus $\mathcal{L}_{5}$ is contained in the field $\mathbb{C}(a, b, z)$, where $F_{4}(z)=0$ (and $a, b$ are algebraically independent). The $S_{3}$ action from Theorem 1 extends naturally to the field $\mathbb{C}(a, b, z)$ because the action of $S_{3}$ is $\varphi$-equivariant. It is generated by the transformations

$$
\sigma:(a, b, z) \mapsto\left(\frac{a}{b}, \frac{1}{b}, \frac{1}{z}\right), \quad \tau:(a, b, z) \mapsto(a,-a-b-1,1-z) .
$$

Let $H=\langle\sigma, \tau\rangle$. Then, $H \cong S_{3}$. We have the following:

Theorem 3. The function field of $\mathcal{L}_{5}$ is given by $\mathbb{C}\left(\mathcal{L}_{5}\right)=\mathbb{C}(a, b, z)^{H}$. Moreover, the invariants of such action are $u, v$ and $w$ where

$$
w=\frac{\left(z^{2}-z+1\right)^{3}}{z^{2}(z-1)^{2}}
$$

In particular,

$$
\mathbb{C}\left(\mathcal{L}_{5}\right)=\mathbb{C}(u, v, w)
$$

where the equation of $w$ in terms of $u, v$ is

$$
c_{2} w^{2}+c_{1} w+c_{0}=0
$$

with $c_{0}, c_{1}, c_{2}$ as follows:

$$
\begin{aligned}
c_{2}= & 64 v^{2}(u-4 v+1)^{2} \\
c_{1}= & -4 v\left(-272 v^{2} u-20 v u^{2}+2592 v^{3}-4672 v^{2}+4 u^{3}+16 v^{3} u^{2}-15 v u^{4}\right. \\
& \left.-96 v^{2} u^{2}+24 v^{2} u^{3}+2 u^{5}-12 u^{4}+92 v u^{3}+576 v u-128 v^{4}-288 v^{3} u\right) \\
c_{1}= & \left(u^{2}+4 v u+4 v^{2}-48 v\right)^{3}
\end{aligned}
$$

Proof. From definitions of $u$ and $v$ we determine that $[\mathbb{C}(a, b): \mathbb{C}(u, v)]=6$. Then, $[\mathbb{C}(a, b, z): \mathbb{C}(u, v)]=12$. The irreducible polynomial of degree 12 of $z$ over $\mathbb{C}(u, v)$ can be easily determined; see [25 for details. Hence, $\left[\mathbb{C}(a, b, z)^{H}: \mathbb{C}(u, v)\right]=2$. It is easily verified that $u, v$ and $w$ are invariants under the action of $H$. Hence, $\mathbb{C}(u, v, w)$ is a subfield of $\mathbb{C}(a, b, z)^{H}$. It is left to show that $[\mathbb{C}(u, v, w): \mathbb{C}(u, v)]=2$, see Fig. 2, We have the system of equations 


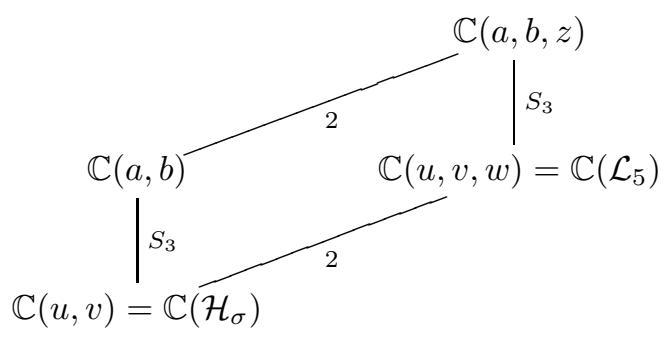

Figure 2. The function field of $\mathcal{L}_{5}$

$$
\left\{\begin{array}{l}
u b(a+b+1)-2 a\left(a b+b^{2}+b+a+1\right)=0 \\
v b(a+b+1)-a^{3}=0 \\
w z^{2}(z-1)^{2}-\left(z^{2}-z+1\right)^{3}=0 \\
(-2 a-1) z^{2}+\left(-2 b+2 a+2 a b+a^{2}\right) z-b^{2}-2 a b=0
\end{array}\right.
$$

Since $\mathbb{C}(a, b) \subset \mathbb{C}(u, v, z)$ we can express $a$ and $b$ as rational functions in $u, v, z$; see [25] for explicit expressions. From the above system we are left with the third equation and the degree 12 polynomial in $z$ with coefficients in $\mathbb{C}(u, v)$. Taking the resultant of these two polynomials with respect to $z$ gives a degree 2 irreducible polynomial of $w$ with coefficients as in Eq. (15). This completes the proof.

3.1. Computing the locus $\mathcal{L}_{5}$. We denote by $J_{2}, J_{4}, J_{6}, J_{10}$ the classical invariants of $C$, for their definitions see 11] or [16. These are homogeneous polynomials (of degree indicated by subscript) in the coefficients of a sextic $f(X, Z)$ defining $C$

$$
Y^{2}=f(X, Z)=a_{6} X^{6}+a_{5} X^{5} Z+\cdots+a_{1} X Z^{5}+a_{0}
$$

and they are a complete set of $S L_{2}(k)$-invariants (acting by coordinate change). They yield homogeneous coordinates on the moduli space $\mathcal{M}_{2}$ (Igusa coordinates). The corresponding inhomogeneous coordinates are the absolute invariants

$$
i_{1}:=144 \frac{J_{4}}{J_{2}^{2}}, \quad i_{2}:=-1728 \frac{J_{2} J_{4}-3 J_{6}}{J_{2}^{3}}, \quad i_{3}:=486 \frac{J_{10}}{J_{2}^{5}}
$$

Two genus 2 curves with $J_{2} \neq 0$ are isomorphic if and only if they have the same absolute invariants.

For a curve in $\mathcal{L}_{5}$ we can express $i_{1}, i_{2}, i_{3}$ in terms of $a, b, z$ by using Eq. (9). In the degenerate cases they only depend on one parameter which we eliminate to obtain an equation in $i_{1}, i_{2}, i_{3}$ defining the corresponding locus in $\mathcal{M}_{2}$; see next section for details.

Since from Eq. (16) we can express $a, b$ as rational functions in $u, v, z$, then $i_{1}, i_{2}, i_{3}$ are given as rational functions in $u, v, z$. By using the definition of $w$ in terms of $z$ we express $i_{1}, i_{2}, i_{3}$ in terms of $u, v$, and $w$. From the equation of $w$ in terms of $u, v$ (this is a degree 2 polynomial in $w$ with coefficients in $\mathbb{C}(u, v)$ ), we eliminate $w$ and are left with three equations

$$
f_{1}\left(i_{1}, u, v\right)=0, \quad f_{2}\left(i_{2}, u, v\right)=0, \quad f_{3}\left(i_{3}, u, v\right)=0 .
$$

Eliminating $u$ and $v$ gives the equation of $\mathcal{L}_{5}$. This equation is available at 25 . 


\section{Degenerate CASES}

Let $\mathcal{Y}_{1}$ (resp., $\mathcal{Y}_{2}$, resp., $\mathcal{Y}_{3}$ ) be the locus in $\mathcal{M}_{2}$ of the genus 2 curves admitting a degree 5 cover $C \rightarrow E$ such that the corresponding cover $\phi: \mathbb{P}^{1} \rightarrow \mathbb{P}^{1}$ is of degenerate type I (resp., II, resp., III). In these cases the cover $\phi$ has only four branch points all of which are ramified in $E$ (in the situation of Fig. (1)). From their ramification structure it follows that in these cases there is a 1-1 correspondence between $\phi$ and $\psi$. In other words, each of the three curves $\mathcal{Y}_{i}$ is isomorphic to the corresponding Hurwitz space. In order to compute that Hurwitz space, we take the equation for $\phi$ from Theorem 1 and symmetrize by the $S_{3}$ action. In cases I and II, the branch point $t=1$ is distinguished from $t=0$ and $t=\infty$ by its ramification structure. Thus, only the action of $\sigma \in S_{3}$ which comes from permuting 0 and $\infty$ has to be considered. That's why cases I and II are easier than case III. We treat them in the present section.

Let $\varphi(x)$ be as in Theorem 1, The corresponding cover $\phi$ belongs to degenerate case I (resp., II), normalized as in Theorem 2, if and only if the following condition I (resp., II) holds.

$$
\begin{gathered}
4 b=a^{2} \\
b=a-1
\end{gathered}
$$

4.1. Case I. For case $\mathbf{I}$, this follows because $\phi^{-1}(1)$ contains a point of ramification index 4 if and only if the two roots of $F_{3}(x)$ collapse. The latter is equivalent to $\operatorname{Disc}\left(F_{3}, x\right)=0$, which yields $b=\frac{a^{2}}{4}$. The argument in case $\mathbf{I I}$ is similar (replacing the condition $\operatorname{Disc}\left(F_{3}, x\right)=0$ by $\left.F_{3}(1)=0\right)$.

We compute that the root $z$ of $F_{4}(x)$ not over $t=1$ equals

$$
\frac{a(8+a)}{4(2 a+1)}
$$

respectively

$$
\frac{(3 a-1)(a-1)}{(2 a+1)}
$$

in case I (resp., II). Thus, from Eq. (9) and Eq. (10) we get that $C$ has an equation of the form

$$
y^{2}=x(x-1)\left(b_{3} x^{3}+b_{2} x^{2}+b_{1} x+b_{0}\right)
$$

where $b_{0}, \ldots b_{3}$ depend only on $a$.

Remark 5. In case I the branch points of $\phi$ are $t=0,1, \infty$ and

$$
\lambda=\varphi(z)=\frac{4(2 a+1)^{3}\left(a^{2}+4 a+8\right)^{2}}{(2-a)^{5}(a+2)^{3}}
$$

Thus, if we fix $\lambda$ then there are 8 corresponding maps $\phi$ up to equivalence. This is consistent with the fact that modulo conjugation there are exactly 8 generating systems $\sigma$ of $S_{5}$ of cycle type $\left((2)^{2},(2)^{2},(4),(2)\right)$ and with product 1 . There is a similar correspondence in case II.

The absolute invariants of $C$ are rational functions in $a$ of degree 24, 36, and 60 respectively. We make the substitution

$$
T:=\left(\frac{a-2}{5(a+2)}\right)^{2} .
$$


Then $i_{1}, i_{2}, i_{3}$ can be expressed as rational functions in $T$ of degree 12, 18, 30 as follows:

$$
\begin{aligned}
i_{1} & =\frac{45}{J_{2}^{2}}\left(200225830078125 T^{12}-1719272460937500 T^{11}+565236035156250 T^{10}\right. \\
& -54100617187500 T^{9}+13999178671875 T^{8}-4261746675000 T^{7}+606825435500 T^{6} \\
& \left.\left.-54844543800 T^{5}+4205965699 T^{4}-236021164 T^{3}+6405914 T^{2}+6116 T-211\right)\right) \\
i_{2} & =\frac{135}{J_{2}^{3}}\left(6335270404815673828125 T^{18}+113021224021911621093750 T^{17}\right. \\
& -137079483776092529296875 T^{16}+35382386975097656250000 T^{15} \\
& -5727170209350585937500 T^{14}+1661335117119140625000 T^{13} \\
& -438672743956054687500 T^{12}+71535083209593750000 T^{11} \\
& -9593401735688906250 T^{10}+1451100945145362500 T^{9}-198805994903162250 T^{8} \\
& +18781404045085680 T^{7}-1082976623440908 T^{6}+34245258932328 T^{5} \\
& \left.-572847931740 T^{4}+10845126800 T^{3}-380189355 T^{2}+11646582 T-3107\right) \\
i_{3} & =\frac{49766400}{J_{2}^{5}} T(9 T-1)^{5}\left(25 T^{2}+6 T+1\right)^{5}(25 T-1)^{7}
\end{aligned}
$$

where

$$
J_{2}=5859375 T^{6}-129843750 T^{5}-31959375 T^{4}-6330100 T^{3}-54927 T^{2}+12506 T-17
$$

Proposition 1. $\mathbb{C}\left(\mathcal{Y}_{1}\right)=\mathbb{C}(T)$

Proof. Computationally, one can solve $T$ from above equations and express it as a rational function in $i_{1}, i_{2}, i_{3}$.

Thus, the map

$$
\begin{gathered}
\Phi: \quad \mathbb{C} \backslash\left\{\Delta_{1} \neq 0\right\} \rightarrow \mathcal{Y}_{1} \\
T \rightarrow\left(i_{1}, i_{2}, i_{3}\right)
\end{gathered}
$$

gives a birational parametrization of $\mathcal{Y}_{1}$. The degrees of the field extensions are as in the following diagram. One can compute equations defining $\mathcal{Y}_{1}$ as a subvariety

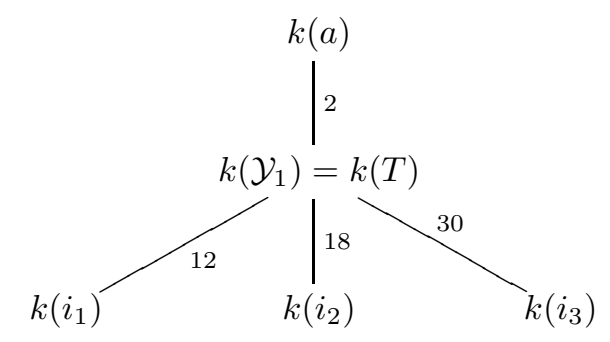

of $\mathcal{M}_{2}$ (i.e., in terms of $\left.i_{1}, i_{2}, i_{3}\right)$ by eliminating $T$. Such equations are large, hence 
we don't display them here. The degrees of this equation in $i_{1}, i_{2}, i_{3}$ can be read from the above diagram.

The elliptic curve $E$ has j-invariant

$j=\frac{\left(11390625 T^{8}+1215000 T^{7}+99900 T^{6}+925032 T^{5}+550 T^{4}+40 T^{3}+380 T^{2}-40 T+1\right)^{3}}{4096 T^{5}(25 T-1)^{2}\left(25 T^{2}+6 T+1\right)^{4}(9 T-1)^{6}}$

To compute $j$ we first write it as a rational function in $a$. Then, we make the substitution $T=\left(\frac{a-2}{5(a+2)}\right)^{2}$.

Remark 6. If $J_{2}=0$, then there are exactly six isomorphism classes of curves. In the moduli space they are described by invariants $a_{1}, a_{2}$ as in [26].

4.1.1. Automorphism groups. Next we want to find if the curves in $\mathcal{Y}_{1}$ have extra automorphisms.

Proposition 2. Let $C$ be a genus 2 curve in the locus $\mathcal{Y}_{1}$. Then, the automorphism group of $C$ is either $\mathbb{Z}_{2}$ or $V_{4}$. Moreover, there are exactly 36 isomorphism classes of curves with automorphism group $V_{4}$ given by the following values for $T$ :

$$
\begin{array}{r}
\left(5625 T^{3}-650 T^{2}+73 T+8\right)\left(1265625 T^{4}-67500 T^{3}+89550 T^{2}+516 T+1\right) \\
\left(625 T^{3}-25 T^{2}-9 T+1\right)\left(5625 T^{5}+18075 T^{4}+8282 T^{3}+918 T^{2}-131 T-1\right) \\
\left(109375 T^{5}+18125 T^{4}-12450 T^{3}+1186 T^{2}-13 T+1\right)\left(7119140625 T^{7}+6391406250 T^{6}\right. \\
\left.+2582859375 T^{5}+476007500 T^{4}+19626975 T^{3}-1411606 T^{2}+257473 T-4096\right) \\
\left(158203125000 T^{9}+85869140625 T^{8}+32415625000 T^{7}+6116187500 T^{6}+74885000 T^{5}\right. \\
\left.-94007050 T^{4}-7398504 T^{3}+1091468 T^{2}+48 T+1\right)=0
\end{array}
$$

Proof. We substitute the expressions for $i_{1}, i_{2}, i_{3}$ in the locus of curves with extra automorphisms given in [26. Using methods developed in [26, it is an easy computational exercise to show that for each $T$ as above, the automorphism group of the corresponding curve is the Klein 4-group. Moreover, the $i_{1}, i_{2}, i_{3}$ as above don't satisfy any of the loci of curves with automorphism group $D_{8}$ or $D_{12}$. If $J_{2}=0$ then we proceed similarly.

Since the above equation has degree 36, then there are at most 36 genus 2 curves corresponding to the above values for $T$. We find the resultant with respect to $T$ of the above equation and the equation which gives $i_{1}$ in terms of $T$. We get a degree 36 polynomial in $i_{1}$ which has nonzero discriminant. Thus, we have 36 distinct values for $i_{1}$ and therefore 36 distinct isomorphism classes of genus 2 curves.

4.2. Case II. The condition $F_{3}(1)=0$ is equivalent to

$$
b=a-1,
$$

which we assume for the rest of this subsection. Then

$$
z=\frac{(3 a-1)(a-1)}{(2 a+1)}
$$

is the root of $F_{4}(x)$ not over $t=1$. Thus, $C$ has equation

$$
y^{2}=x(x-1)\left(b_{3} x^{3}+b_{2} x^{2}+b_{1} x+b_{0}\right)
$$


where

$$
\begin{aligned}
& b_{3}=(2 a+1)(-8+9 a)^{2} \\
& b_{2}=-27 a^{6}-54 a^{5}+468 a^{4}-958 a^{3}+381 a^{2}+400 a-192 \\
& b_{1}=-18 a^{6}+380 a^{5}-1000 a^{4}+726 a^{3}+499 a^{2}-752 a+192 \\
& b_{0}=(a+8)^{2}(a-1)^{3}(3 a-1),
\end{aligned}
$$

where

$$
a(9 a-8)(a+8)(2 a+1)(3 a-1)(a-1)(a-2) \neq 0 .
$$

This condition is obtained by substituting $b=a-1$ in $\Delta(a, b)$. Summarizing we have the following:

Proposition 3. Let $C$ be a genus 2 curve in the locus $\mathcal{Y}_{2}$. Then $C$ is given by

$$
y^{2}=x(x-1)\left(b_{3} x^{3}+b_{2} x^{2}+b_{1} x+b_{0}\right)
$$

where $b_{0}, b_{1}, b_{2}, b_{3}$ are as in Eq. (19). Moreover, the equation of $E$ is

$$
s^{2}=t(t-1)\left(t-\frac{(3 a-1)^{3}(a+8)^{2}(a-1)}{27 a(a-2)^{5}}\right)
$$

The proof is as for Case I).

Remark 7. We see that if we fix the 4 branch points of $\phi$, then there are 6 corresponding covers $\phi$. Again this is consistent with the fact that there are 6 classes of generating systems of $S_{5}$ of cycle structure $\left((2)^{2},(2)^{2},(2) \cdot(3),(2)\right)$ and product 1.

The absolute invariants $i_{1}, i_{2}, i_{3}$ of $C$ are rational functions in

$$
T:=\left(\frac{a}{a-2}\right)^{2}
$$

of degree 8,12 , and 20 as follows:

$$
\begin{aligned}
i_{1}= & -\frac{9}{J_{2}^{2}}\left(1953125 T^{8}+100859375 T^{7}-133684375 T^{6}-17761750 T^{5}+60906155 T^{4}\right. \\
& \left.-14020705 T^{3}+115631 T^{2}-46816 T-256\right) \\
i_{2}= & -27 \\
8 J_{2}^{3} & \left(96435546875 T^{12}-4709765625000 T^{11}+10970742187500 T^{10}+4833343750000 T^{9}\right. \\
& -31399133343750 T^{8}+30923034102000 T^{7}-13348926086820 T^{6}+3049853644080 T^{5} \\
& \left.-409782059325 T^{4}+10407596440 T^{3}+1223394432 T^{2}-18880512 T+32768\right) \\
i_{3}= & -\frac{59049}{4096 J_{2}^{5}} T^{2}(25 T-1)^{5}(25 T-16)^{5}(T-1)^{7}
\end{aligned}
$$

where $J_{2}$ is

$$
J_{2}=4375 T^{4}-12850 T^{3}+11457 T^{2}+458 T+43
$$

Proposition 4. $\mathbb{C}\left(\mathcal{Y}_{2}\right)=\mathbb{C}(T)$

Proof. $T$ can be eliminated from the above expressions and expressed as a rational function in $i_{1}, i_{2}, i_{3}$. 
The elliptic curve $E$ associated with $C$ has j-invariant $j=\frac{\left(9765625 T^{6}-23437500 T^{5}+19218750 T^{4}-6087500 T^{3}+560625 T^{2}+166368 T+256\right)^{3}}{729 T(T-1)^{2}(25 T-16)^{4}(25 T-1)^{6}}$ To compute $j$ one proceeds similarly as in Case I).

\subsubsection{Automorphism groups.}

Proposition 5. Let $C$ be a genus 2 curve in the locus $\mathcal{Y}_{2}$. Then, the automorphism group of $C$ is either $\mathbb{Z}_{2}$ or $V_{4}$. Moreover, there are exactly 25 isomorphism classes of curves with automorphism group $V_{4}$ given by the following values for $T$ :

$$
\begin{gathered}
\left(25 T^{2}+34 T+13\right)\left(25 T^{2}-26 T+10\right)\left(15625 T^{3}-3750 T^{2}-6075 T+32\right) \\
\left(225 T^{3}-634 T^{2}-151 T-16\right)\left(625 T^{4}-800 T^{3}+156 T^{2}+74 T-1\right) \\
\left(625 T^{5}+22325 T^{4}+892131 T^{3}-338857 T^{2}+48160 T-2304\right)\left(421875 T^{6}+\right. \\
\left.2402500 T^{5}-6942350 T^{4}+5673748 T^{3}-1488397 T^{2}-20464 T-256\right)=0
\end{gathered}
$$

The proof is computational and similar to the one in Case I.

4.3. Case III). Let $C$ be a genus 2 curve admitting a degree 5 cover $C \rightarrow E$ such that the corresponding cover $\phi: \mathbb{P}^{1} \rightarrow \mathbb{P}^{1}$ has ramification structure

$$
(2)^{2},(2)^{2},(2)^{2},(3)
$$

Denote the locus of such curves in $\mathcal{M}_{2}$ by $\mathcal{Y}_{3}$. This case is obtained when the ramified points over the last two branch points coalesce, i.e., $F_{4}(x)$ has a double root. We compute

$$
\operatorname{Disc}\left(F_{4}(x)\right)=a\left(a^{3}+4 b a^{2}+4 a^{2}-12 b a+4 a b^{2}+4 a-16 b-16 b^{2}\right)
$$

If $a=0$ then $\varphi(x)=x$, a forbidden case. Let $\overline{\mathcal{Y}}_{3}$ denote the genus 1 curve

$$
\mathcal{Y}_{3}: \quad a^{3}+4 b a^{2}+4 a^{2}-12 b a+4 a b^{2}+4 a-16 b-16 b^{2}=0
$$

with $j$-invariant $j=\frac{702595369}{72900}$.

The equation of $C$ is given by

$$
y^{2}=x(x-1)\left(x-\frac{a^{2}+2 a b+2 a-2 b}{2(2 a+1)}\right)\left(x^{2}-x-\frac{3 a\left(a^{2}-4\right)}{4(2 a+1)(a-4)}\right)
$$

such that

(23) $a\left(a^{2}-4\right)(2 a+1)\left(3 a^{3}-12 a-1\right)(a-4)\left(96 a^{5}-400 a^{4}-128 a^{3}+800 a^{2}-72 a-225\right) \neq 0$

The elliptic curve $E$ has equation

$$
s^{2}=t(t-1)(t-\lambda)
$$

where $\lambda=\varphi(z)$ and

$$
z=\frac{a^{2}+2 a b+2 a-2 b}{2(2 a+1)}
$$

We don't display $\lambda$ here but it is easily computable; see [25].

Equations defining $\mathcal{Y}_{3}$ : Let $u$ and $v$ be the $S_{3}$ - invariants. The condition Eq. (21) is equivalent to

$$
2 u+v-16=0 .
$$


Denote this genus 0 curve by $\mathcal{Y}_{3}^{\prime}$. This implies that the discriminant of the field extension $\mathbb{C}(u, v, w) / \mathbb{C}(u, v)$

$\Delta_{w}=16(v-16+2 u)\left(2 u^{3}+u^{2} v-36 u v-16 v^{2}-108 v\right)(u-4 v-2)^{2}\left(16 v-4 u v+u^{2}\right)^{2} v^{2}$ is $\Delta_{w}=0$. Hence, $[\mathbb{C}(u, v, w): \mathbb{C}(u, v)]=1$ and

$$
\mathbb{C}(u, v, w)=\mathbb{C}(u, v) .
$$

The absolute invariants $i_{1}, i_{2}, i_{3}$ of $C$ can now again be expressed in terms of $u, v$. We have the maps

$$
\begin{aligned}
\overline{\mathcal{Y}}_{3} & \stackrel{\Upsilon}{\rightarrow} \mathcal{Y}_{3}^{\prime} \stackrel{\Psi}{\rightarrow} \mathcal{M}_{2} \\
(a, b) & \rightarrow(u, v) \rightarrow\left(i_{1}, i_{2}, i_{3}\right)
\end{aligned}
$$

such that the image $\Psi\left(\mathcal{Y}_{3}^{\prime}\right)=\mathcal{Y}_{3}$. We know that deg $\Upsilon=6$ and as we see next $\operatorname{deg} \Psi=1$.

Proposition 6. The function field of $\mathcal{Y}_{3}$ is

$$
\mathbb{C}\left(\mathcal{Y}_{3}\right)=\mathbb{C}(u, v) .
$$

Moreover, $u$ and $v$ can be computed explicitly in terms of the absolute invariants $i_{1}, i_{2}, i_{3}$.

Proof. The first part follows from Eq. (25). The second part is computational. Let $i_{j}=\frac{p_{j}(u, v)}{q_{j}(u, v)}$ for $j=1,2,3$, where $p_{i}(u, v), q_{i}(u, v)$ are polynomials in $u, v$. Then, we have the system

$$
\left\{\begin{array}{l}
2 u+v-16=0 \\
i_{1} \cdot q_{1}(u, v)-p_{1}(u, v)=0 \\
i_{2} \cdot q_{2}(u, v)-p_{2}(u, v)=0 \\
i_{3} \cdot q_{3}(u, v)-p_{3}(u, v)=0
\end{array}\right.
$$

One can solve for $u, v$ the system (27) and get $u, v$ as rational functions in $i_{1}, i_{2}, i_{3}$.

Eliminating $u, u$ from the system (27) yields equations defining $\mathcal{Y}_{3}$ as a subvariety of $\mathcal{M}_{2}$. However, such equations are large and we don't display them. For example, $\mathcal{Y}_{3}$ is given as

$$
G\left(i_{1}, i_{2}\right)=0
$$

where $G\left(i_{1}, i_{2}\right)$ is a polynomial in $i_{1}, i_{2}$ of degree 96 and 64 respectively. An equation of $\mathcal{Y}_{3}$ in terms of $i_{2}, i_{3}$ has degrees 160 and 96 respectively; see [25] for details.

Remark 8. The reader who is interested in such equations can use any computational algebra package such as Maple, Magma etc and eliminate u, $v$ via resultants. We used MAPLE 9 and computations sometimes took several days.

\subsubsection{Automorphism groups.}

Proposition 7. Let $C$ be a genus 2 curve in the locus $\mathcal{Y}_{3}$. Then the automorphism group of $C$ is either $\mathbb{Z}_{2}$ or $V_{4}$. Moreover, there are 138 isomorphism classes of curves with automorphism group $V_{4}$ given by the following values for a: 
(28)

$$
\begin{array}{r}
\left(24 a^{5}-84 a^{4}-144 a^{3}+328 a^{2}+220 a+17\right)\left(96 a^{5}-400 a^{4}-131 a^{3}+800 a^{2}-60 a-224\right)^{2} \\
\left(72 a^{5}-316 a^{4}+16 a^{3}+472 a^{2}-292 a-241\right)^{2}\left(9 a^{6}-72 a^{4}-6 a^{3}+152 a^{2}-4 a-15\right) \\
\left(9216 a^{10}-76800 a^{9}+136000 a^{8}+252832 a^{7}-634615 a^{6}-184640 a^{5}+824616 a^{4}-57222 a^{3}\right. \\
\left.-340360 a^{2}+30348 a+47025\right)\left(216 a^{11}+1548 a^{10}-20688 a^{9}+25776 a^{8}+133824 a^{7}\right. \\
\left.-190976 a^{6}-286296 a^{5}+289508 a^{4}+231440 a^{3}-65056 a^{2}-58032 a-6975\right)\left(27648 a^{13}\right. \\
-230400 a^{12}+295680 a^{11}+1689600 a^{10}-3531264 a^{9}-3711808 a^{8}+10386272 a^{7} \\
\left.+2095872 a^{6}-11895424 a^{5}+1027312 a^{4}+5156035 a^{3}-398800 a^{2}-849036 a-61696\right) \\
\left(648 a^{14}+4644 a^{13}-64656 a^{12}+58320 a^{11}+658152 a^{10}-935328 a^{9}-2364128 a^{8}\right. \\
+3266608 a^{7}+3718976 a^{6}-3536792 a^{5}-2448532 a^{4}+439027 a^{3}+404320 a^{2} \\
+174132 a+57600)=0
\end{array}
$$

Proof. We substitute the expressions for $i_{1}, i_{2}, i_{3}$ in the locus of curves with extra automorphisms given in [26]. Thus, all genus 2 curves obtained by the above values of $a$ have $V_{4}$ embedded in their automorphism group. Moreover, for each $a$ as above, the automorphism group of the corresponding curve is not $D_{8}, D_{12}$. Hence, it is Klein 4-group. If $J_{2}=0$ then we proceed similarly.

The invariant $i_{1}=\frac{p(u, v)}{q(u, v)}$, where $p(u, v), q(u, v)$ are polynomials in $u, v$. We take the resultant of $i_{1} q(u, v)-p(u, v)$ and $\operatorname{Disc} F_{4}(x)$ with respect to $b$. The result is a polynomial in $i_{1}$ and $a$ of degree 2 and 69 respectively. Taking the resultant of this polynomial and the polynomial of degree 69 in Eq. (28) we get a polynomial in $i_{1}$ of degree 138 . The discriminant of this polynomial is nonzero. Hence, there are 138 isomorphism classes of genus 2 curves. This is to be expected since for each $a$ we have two values of $b$ determined from Eq. (21).

\section{Concluding REMARKS}

The main goal of this paper is to compute an equation for the locus $\mathcal{L}_{5}$ and its subloci. This equation is in terms of the Igusa coordinates $i_{1}, i_{2}, i_{3}$ on the moduli space of genus 2 curves. That $\mathcal{L}_{5}$ is a rational variety follows also from the general theory of "diagonal modular surfaces", see Kani [13]. The computations performed give us precise information on the locus $\mathcal{L}_{5}$ and its degenerate subloci which would be difficult to obtain with other methods. Since the equations describing $\mathcal{L}_{5}$ and its subloci are big and take several pages to display we chose not to display them. Instead, we only gave birational parametrizations of these spaces.

For the reader who wants to use such equations but doesn't want to go through the lengthy computations of obtaining them we can provide them; see [25].

Degree $n$ covers $\psi: C \rightarrow E$ have been successfully used in number theory applications. The genus 2 curve with the largest known number of rational points has automorphism group isomorphic to $D_{12}$; thus it has degree 2 cover to an elliptic curve. It was found by Keller and Kulesz and it is known to have at least 588 rational points; see [18]. Using degree $n=2,3$ covers $\psi: C \rightarrow E$ Howe, Leprevost, and Poonen [9] were able to construct a family of genus 2 curves whose Jacobians 
each have large rational torsion subgroups. Using formulas in Theorem 1 and 2, similar techniques probably could be applied using degree $n \geq 5$ covers.

The moduli spaces $\mathcal{L}_{n}$ also have applications to the study of integrable systems. The interested reader should check [1 for a complete survey on this topic. The authors describe the moduli space of genus two curves that admit a degree $n$ elliptic subcover in several ways: by algebra, group theory, monodromy, and topology. We hope this paper fills the gap in the literature of explicitly describing such moduli spaces as subspaces of the moduli space $\mathcal{M}_{2}$.

\section{REFERENCES}

[1] R. Accola, E. Previato, Covers of tori: genus two. Lett. Math. Phys. 76 (2006), no. 2-3, $135-161$.

[2] C. Birkenhake, H. Wilhelm, Humbert surfaces and the Kummer plane. Trans. Amer. Math. Soc. 355 (2003), no. 5, 1819-1841.

[3] G. Frey, On elliptic curves with isomorphic torsion structures and corresponding curves of genus 2. Elliptic curves, modular forms, and Fermat's last theorem (Hong Kong, 1993), 79-98, Ser. Number Theory, I, Internat. Press, Cambridge, MA, 1995.

[4] G. Frey and E. Kani, Curves of genus 2 covering elliptic curves and an arithmetic application. Arithmetic algebraic geometry (Texel, 1989), 153-176, Progr. Math., 89, Birkhäuser Boston, MA, 1991.

[5] G. Frey, E. Kani, H. Völklein, Curves of genus 2 with elliptic differentials and associated Hurwitz spaces, preprint 2004.

[6] M. Fried And H. VölkLein, The inverse Galois problem and rational points on moduli spaces, Math. Annalen 290 (1991) , 771-800.

[7] G. van der Geer, Hilbert modular surfaces, Springer, Berlin, 1987.

[8] J. Gutierrez and T. Shaska, Hyperelliptic curves with extra involutions, LMS J. of Comp. Math., 8, (2005), 102-115.

[9] E. Howe, F. Leprévost, and B. Poonen, Large torsion subgroups of split Jacobians of curves of genus two or three. Forum. Math, 12 (2000), no. 3, 315-364.

[10] G. Humbert Sur les fonctionnes abliennes singulires. I, II, III. J. Math. Pures Appl. serie 5, t. V, 233-350 (1899); t. VI, 279-386 (1900); t. VII, 97-123 (1901).

[11] J. IgusA, Arithmetic Variety Moduli for genus 2. Ann. of Math. (2), 72, 612-649, 1960.

[12] E. Kani, The number of curves of genus two with elliptic differentials. J. Reine Angew. Math. 485 (1997), 93-121.

[13] E, Kani And W. Schanz, Diagonal quotient surfaces. Manuscripta Math. 93, no. 1, 67-108, 1997.

[14] E, Kani And W. Schanz, Modular diagonal quotient surfaces. Math. Z. 227 (1998), no. 2, $337-366$.

[15] A. Krazer, Lehrbuch der Thetafunctionen, Chelsea, New York, 1970.

[16] V. Krishnamorthy, T. Shaska, H. Voelklein, Invariants of binary forms, Developments in Mathematics, Kluwer Academic Publishers, Boston, MA, 2004, 101-122.

[17] M. R. Kunn, Curves of genus 2 with split Jacobian. Trans. Amer. Math. Soc 307, 41-49, 1988.

[18] W. Keller, L. Kulesz, Courbes algbriques de genre 2 et 3 possdant de nombreux points rationnels. C. R. Acad. Sci. Paris Sr. I Math. 321 (1995), no. 11, 1469-1472.

[19] K. Magaard, S. Shpectorov and H. Voelklein, A GAP package for braid orbit computation, and applications, Experimental Math., 12 (2003), no. 4, 385-393.

[20] Maple 9, Waterloo Maple Inc., 2002.

[21] N. Murabayashi, The moduli space of curves of genus two covering elliptic curves. Manuscripta Math. 84 (1994), no. 2, 125-133.

[22] T. Shaska, Genus 2 curves with (n, n)-decomposable Jacobians, Jour. Symb. Comp., Vol 31, no. 5, pg. 603-617, 2001.

[23] T. Shaska, Genus 2 curves with degree 3 elliptic subcovers, Forum. Math., vol. 16, 2, pg. 263-280, 2004.

[24] T. Shaska, Genus two curves covering elliptic curves: a computational approach. Computational aspects of algebraic curves, Lecture Notes Ser. Comput., 13, 2005, 206-231. 
[25] http://www. oakland.edu/ ${ }^{\sim}$ shaska

[26] T. Shaska AND H. Völklein, Elliptic subfields and automorphisms of genus two fields, Algebra, Arithmetic and Geometry with Applications. Papers from Shreeram S. Abhyankar's 70th Birthday Conference (West Lafayette, 2000), pg. 687 - 707, Springer (2004).

[27] G. Tamme, Ein Satz über hyperelliptische Funktionenkörper. J. Reine Angew. Math. 257, 217-220, 1972.

[28] H. VölkLein, Groups as Galois Groups - an Introduction, Cambr. Studies in Adv. Math. 53, Cambridge Univ. Press, 1996.

Department of Mathematics, Wayne State University, Detroit, Mi 48201, USA

E-mail address: kaym@math.wayne.edu

Department of Mathematics, Oakland University, Rochester, Mi 48309, USA

E-mail address: shaska@oakland.edu

Institut für Experimentelle Mathematik, Universität Duisburg-Essen, Ellernstr. 29, D-45326 Essen, Germany

E-mail address: voelkle@iem.uni-due.de 